\title{
Adaptive Dynamics of Speciation: Ecological Underpinnings
}

Stefan A.H. Geritz (stefan.geritz@utu.fi)

Éva Kisdi (evakis@utu.fi)

Géza Meszéna (geza.meszena@elte.ru)

Johan A.J. Metz (metz@rulsfb.leidenuniv.nl)

\section{Approved by}

Leen Hordijk (hordijk@iiasa.ac.at)

Director, IIASA

November 2004 


\section{Contents}

1 Introduction 1

2 Invasion Fitness 2

3 Phenotypic Evolution by Trait Substitution 4

4 The Emergence of Diversity: Evolutionary Branching 5

5 Evolutionary Branching and Speciation 9

6 Adaptive Dynamics: Alternative Approaches 15

7 Concluding Comments 16 


\author{
About the Authors \\ Stefan A.H. Geritz \\ Department of Mathematical Sciences \\ University of Turku \\ FIN-20014 Turku, Finland \\ Éva Kisdi \\ Department of Mathematical Sciences \\ University of Turku \\ FIN-20014 Turku, Finland \\ Géza Meszéna \\ Department of Biological Physics \\ Eötvös University \\ Pazmany Peter setany 1A \\ H-1117 Budapest \\ Hungary \\ Johan A.J. Metz \\ Section Theoretical Biology \\ University of Leiden \\ Kaiserstraat 63, NL-2311 GP Leiden, The Netherlands \\ and \\ Adaptive Dynamics Network \\ International Institute for Applied Systems Analysis \\ A-2361 Laxenburg, Austria
}

\title{
Acknowledgments
}

This work was supported by grants from the Academy of Finland, from the Turku University Foundation, from the Hungarian Science Foundation (OTKA T 019272), from the Hungarian Ministry of Education (FKFP 0187/1999), and from the Dutch Science Foundation (NWO 048-011-039). Additional support was provided by the European Research Training Network ModLife (Modern Life-History Theory and its Application to the Management of Natural Resources), funded through the Human Potential Programme of the European Commission (Contract HPRN-CT-2000-00051). 


\title{
Adaptive Dynamics of Speciation: Ecological Underpinnings
}

\author{
Stefan A.H. Geritz \\ Éva Kisdi \\ Géza Meszéna \\ Johan A.J. Metz
}

\section{Introduction}

Speciation occurs when a population splits into ecologically differentiated and reproductively isolated lineages. In this chapter, we focus on the ecological side of nonallopatric speciation: Under what ecological conditions is speciation promoted by natural selection? What are the appropriate tools to identify speciation-prone ecological systems?

For speciation to occur, a population must have the potential to become polymorphic (i.e., it must harbor heritable variation). Moreover, this variation must be under disruptive selection that favors extreme phenotypes at the cost of intermediate ones. With disruptive selection, a genetic polymorphism can be stable only if selection is frequency dependent (Pimm 1979; see Chapter 3 in Dieckmann et al. 2004). Some appropriate form of frequency dependence is thus an ecological prerequisite for nonallopatric speciation.

Frequency-dependent selection is ubiquitous in nature. It occurs, among many other examples, in the context of resource competition (Christiansen and Loeschcke 1980; see Box 1), predatorprey systems (Marrow et al. 1992), multiple habitats (Levene 1953), stochastic environments (Kisdi and Meszéna 1993; Chesson 1994), asymmetric competition (Maynard Smith and Brown 1986), mutualistic interactions (Law and Dieckmann 1998), and behavioral conflicts (Maynard Smith and Price 1973; Hofbauer and Sigmund 1990).

The theory of adaptive dynamics is a framework devised to model the evolution of continuous traits driven by frequency-dependent selection. It can be applied to various ecological settings and is particularly suitable for incorporating ecological complexity. The adaptive dynamic analysis reveals the course of long-term evolution expected in a given ecological scenario and, in particular, shows whether, and under which conditions, a population is expected to evolve toward a state in which disruptive selection arises and promotes speciation. To achieve analytical tractability in ecologically complex models, many adaptive dynamic models (and much of this chapter) suppress genetic complexity with the assumption of clonally reproducing phenotypes (also referred to as strategies or traits). This enables the efficient identification of interesting features of the engendered selective pressures that deserve further analysis from a genetic perspective.

The analysis begins with the definition of admissible values of the evolving traits (including all trade-offs between traits and other constraints upon them), and the construction of a population dynamic model that incorporates the specific ecological conditions to be investigated, along with a specification of how the model parameters depend on the trait values. From the population dynamic model, one can derive the fitness of any possible rare mutant in a given resident population. 


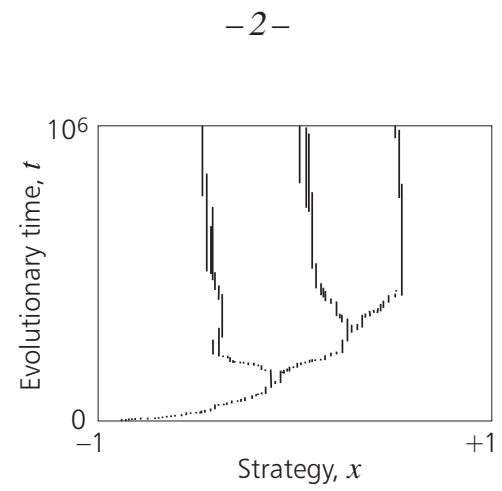

Figure 1 Simulated evolutionary tree for the model described in Box 1 with $r=1, K(x)=(1-$ $\left.x^{2}\right)_{+}, a\left(x, x^{\prime}\right)=\exp \left(-\frac{1}{2}\left(x-x^{\prime}\right)^{2} / \sigma_{a}^{2}\right)$ with $\sigma_{a}=0.35$. The strategy axis (horizontal) is in arbitrary units; the evolutionary time axis is in units of $r^{-1}$. For details of the simulation, see Geritz et al. (1999) or Kisdi and Geritz (1999).

It is thus possible to deduce which mutants can invade the population, and in which direction evolution will proceed via a sequence of successive invasion and fixation events.

Eventually, directional evolution may arrive at a particular trait value for which a successful invading mutant does not oust and replace the former resident; instead, the mutant and the resident coexist. If the two strategies coexist, and if selection in the newly formed dimorphic population is disruptive (i.e., if it favors new mutants that are more extreme and suppresses strategies between those of the two residents), then the clonal population undergoes evolutionary branching, whereby the single initial strategy is replaced by two strategies separated by a gradually widening gap. Figure 1 shows a simulated evolutionary tree with two such branching events. With small mutations, such a split can occur when directional evolution approaches a particular trait value called a branching point.

Evolutionary branching of clonal strategies cannot be equated with speciation, since clonal models of adaptive dynamics are unable to address the question of reproductive isolation. Chapter 5 in Dieckmann et al. 2004 discusses adaptive dynamics with multilocus genetics and the emergence of reproductive isolation during evolutionary branching. Yet, evolutionary branching itself signals that adaptive speciation is promoted by selection in the ecological system considered.

In this chapter we outline one particular framework of adaptive dynamics that has been developed by Metz et al. (1996), Geritz et al. (1997, 1998), and, for directional evolution, Dieckmann and Law (1996). This framework integrates concepts from the modern theory of evolutionarily stable strategies (Maynard Smith 1982; Eshel 1983; Taylor 1989; Nowak 1990; Christiansen 1991) and accommodates evolutionary branching. We constrain this summary mainly to a simple graphic approach; the corresponding analytical treatment (which is indispensable if the theory is to be applied to multidimensional traits or to polymorphic populations that cannot be depicted in simple one- or two-dimensional plots; see Box 5) can be found in Metz et al. (1996) and Geritz et al. (1998).

\section{Invasion Fitness}

Invasion fitness is the exponential growth rate of a rare mutant strategy in the environment set by a given resident population (Metz et al. 1992). The calculation of invasion fitness depends on the particular ecological setting to be investigated. Here we sketch the basics of fitness calculations common to all models.

Consider a large and well-mixed population in which a rare mutant strategy appears. The change in the density of mutants can be described by

$$
n(t+1)=A(E(t)) n(t) .
$$


Box 1 Invasion fitness in a model of competition for a continuous resource

Consider the Lotka-Volterra competition model

$$
\frac{1}{n_{i}} \frac{\mathrm{d} n_{i}}{\mathrm{~d} t}=r\left[1-\frac{\sum_{j} a\left(x_{i}, x_{j}\right) n_{j}}{K\left(x_{i}\right)}\right],
$$

where the trait value $x_{i}$ determines which part of a resource continuum the $i$ th strategy can utilize efficiently (e.g., beak size determines which seeds of a continuous distribution of seed sizes are consumed). The more similar two strategies are, the more their resources overlap, and the more intense the competition. This can be expressed by the commonly used Gaussian competition function $a\left(x_{i}, x_{j}\right)=\exp \left(-\frac{1}{2}\left(x_{i}-x_{j}\right)^{2} / \sigma_{a}^{2}\right)$ (see Christiansen and Fenchel 1977). We assume that the intrinsic growth rate $r$ is constant and that the carrying capacity $K$ is unimodal with a maximum at $x_{0} ; K$ is given by $K(x)=\left(K_{0}-\lambda\left(x-x_{0}\right)^{2}\right)_{+}$, where $(\ldots)_{+}$indicates that negative values are set to zero. This model (or a very similar model) has been investigated, for example, by Christiansen and Loeschcke (1980), Slatkin (1980), Taper and Case (1985), Vincent et al. (1993), Metz et al. (1996), Doebeli (1996b), Dieckmann and Doebeli (1999), Drossel and McKane (1999), Day (2000), and Doebeli and Dieckmann (2000).

As long as a mutant strategy is rare, its self-competition and impact on the resident strategies are negligible. The density of a rare mutant strategy $x^{\prime}$ thus increases exponentially according to

$$
\frac{1}{n^{\prime}} \frac{\mathrm{d} n^{\prime}}{\mathrm{d} t}=r\left[1-\frac{\sum_{j} a\left(x^{\prime}, x_{j}\right) \hat{n}_{j}}{K\left(x^{\prime}\right)}\right],
$$

where $\hat{n}_{j}$ is the equilibrium density of the $j$ th resident. These equilibrium densities can be obtained by setting Equation (a) equal to zero and solving for $n_{i}$. The right-hand side of Equation (b) is the exponential growth rate, or invasion fitness, of the mutant $x^{\prime}$ in a resident population with strategies $x_{1}, \ldots, x_{n}$. Specifically, in a monomorphic resident population with strategy $x$, the equilibrium density is $K(x)$ and the mutant's fitness simplifies to

$$
f\left(x^{\prime}, x\right)=r\left[1-a\left(x^{\prime}, x\right) \frac{K(x)}{K\left(x^{\prime}\right)}\right] .
$$

Figures 1 and 2, and the figure in Box 5, are based on this model.

Here $n$ is the density of mutants or, in structured populations, the vector that contains the density of mutants in various age or stage classes. The matrix $A$ describes population growth as well as transitions between different age or stage classes (Caswell 1989); in an unstructured population, $A$ is simply the annual growth rate. In continuous time, the population growth of the mutant can be described by

$$
\frac{\mathrm{d} n(t)}{\mathrm{d} t}=B(E(t)) n(t) .
$$

The dynamics of the mutant population as specified by $A(E)$ (in discrete time) or $B(E)$ (in continuous time) depends on the properties of the mutant and on the environment $E$. The environment contains all factors that influence population growth, including the abundance of limiting resources, the density of predators or parasites, and abiotic factors. Most importantly, $E$ contains all the effects the resident population has directly or indirectly on the mutant; generally, $E$ depends on the population density of the residents. As long as the mutant is rare, its effect on the environment is negligible.

The exponential growth rate, or invasion fitness, of the mutant strategy is defined by comparing the total density $N(t)$ of mutants, after a sufficiently long time, with the initial density $N(0)$, while keeping the mutant's environment fixed. In structured populations $N$ is the sum of the vector components of $n$, whereas in unstructured populations there is no difference between the 
two. Formally, the invasion fitness is given by (Metz et al. 1992)

$$
f=\lim _{t \rightarrow \infty} \frac{1}{t} \ln \frac{N(t)}{N(0)} .
$$

The long time interval is taken to ensure that the population experiences a representative time series of the possibly fluctuating environment $E(t)$, and that a structured mutant population attains its stationary distribution. For a nonstructured population in a stable environment (which requires a stable resident population), there is no need to consider a long time interval: the invasion fitness of the mutant is then simply $f=\ln A(\hat{E})$ in discrete time and $f=B(\hat{E})$ in continuous time, with $\hat{E}$ being the environment as set by the equilibrium resident population. A positive value of $f$ indicates that the mutant strategy can spread in the population, whereas a mutant with negative $f$ will die out. Box 1 contains an example of how to calculate $f$ for a concrete model.

At the very beginning of the invasion process, typically only a few mutant individuals are present. As a consequence, demographic stochasticity plays an important role so that the mutant may die out despite having a positive invasion fitness $f$. However, the mutant has a positive probability of escaping random extinction whenever its growth rate $f$ is positive (Crow and Kimura 1970; Goel and Richter-Dyn 1974; Dieckmann and Law 1996). Once the mutant has grown sufficiently in number so that demographic stochasticity can be neglected, its further invasion dynamics is given by Equation (1) as long as it is still rare in frequency. Equation (1) ceases to hold once the mutant becomes sufficiently common that it appreciably influences the environment $E$.

Henceforth the fitness of a rare mutant strategy with trait value $x^{\prime}$ in a resident population of strategy $x$ is denoted by $f\left(x^{\prime}, x\right)$ to emphasize that the fitness of a rare mutant depends on its own strategy as well as on the resident strategy, since the latter influences the environment $E$. This notation suppresses the associated ecological variables, such as the equilibrium density of the residents. It is essential to realize, however, that the fitness function $f\left(x^{\prime}, x\right)$ is derived from a population dynamic model that appropriately incorporates the ecological features of the system under study.

\section{Phenotypic Evolution by Trait Substitution}

A single evolutionary step is made when a new strategy invades the population and ousts the former resident. The phenotypes that prevail in the population evolve by a sequence of invasions and substitutions. We assume that mutations occur infrequently, so that the previously invading mutant becomes established and the population reaches its population dynamic equilibrium (in a deterministic or statistical sense) by the time the next mutant arrives, and also that mutations are of small phenotypic effect (i.e., that a mutant strategy is near the resident strategy from which it originated).

Consider a monomorphic resident population with a single strategy $x$. A mutant strategy $x^{\prime}$ can invade this population if its fitness $f\left(x^{\prime}, x\right)$ is positive. If strategy $x$ has a negative fitness when strategy $x^{\prime}$ is already widespread, then the mutant strategy $x^{\prime}$ can eliminate the original resident. We assume that there is no unprotected polymorphism and thus infer that strategy $x^{\prime}$ can replace strategy $x$ if and only if $f\left(x^{\prime}, x\right)$ is positive and $f\left(x, x^{\prime}\right)$ is negative. On the other hand, if both strategies spread when rare, that is, if both $f\left(x^{\prime}, x\right)$ and $f\left(x, x^{\prime}\right)$ are positive, then the two strategies form a protected dimorphism.

In the remainder of this section, as well as in Section 4, we focus on the evolution of strategies specified by a single quantitative trait in monomorphic resident populations. To visualize the course of phenotypic evolution it is useful to depict graphically those mutant strategies that can invade in various resident populations and those strategy pairs that can form protected dimorphisms. Figure 2a shows a so-called pairwise invasibility plot (Matsuda 1985; Van Tienderen and de Jong 

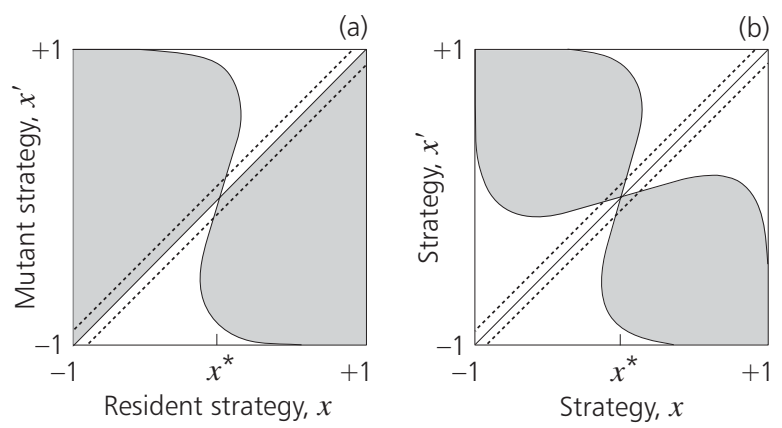

Figure 2 Course of phenotypic evolution for the model described in Box 1 with $r=1, K(x)=(1-$ $\left.x^{2}\right)_{+}, a\left(x, x^{\prime}\right)=\exp \left(-\frac{1}{2}\left(x-x^{\prime}\right)^{2} / \sigma_{a}^{2}\right)$ with $\sigma_{a}=0.5$. (a) Pairwise invasibility plot. Gray areas indicate combinations of mutant strategies $x^{\prime}$ and resident strategies $x$ for which the mutant's fitness $f\left(x^{\prime}, x\right)$ is positive; white areas correspond to strategy combinations such that $f\left(x^{\prime}, x\right)$ is negative. (b) The set of potentially coexisting strategies. Gray areas indicate strategy combinations for which both $f\left(x^{\prime}, x\right)$ and $f\left(x, x^{\prime}\right)$ are positive; protected coexistence outside the gray areas is not possible. In both (a) and (b), the dotted lines schematically illustrate the narrow band of mutants near the resident that can arise by mutations of small phenotypic effect. The singular strategy is denoted by $x^{*}$.

1986): each point inside the gray area represents a resident-mutant strategy combination such that the mutant can invade the population of the resident. Points inside the white area correspond to mutant-resident strategy pairs such that the mutant cannot invade. A pairwise invasibility plot is constructed by evaluating the mutant's fitness $f\left(x^{\prime}, x\right)$ for all values of $x$ and $x^{\prime}$ and "coloring" the corresponding point of the plot according to whether $f\left(x^{\prime}, x\right)$ is positive or negative. In Figure $2 \mathrm{~b}$ the gray area indicates that both $f\left(x^{\prime}, x\right)$ and $f\left(x, x^{\prime}\right)$ are positive, and hence the two strategies are able to coexist. This plot is obtained by first mirroring the pairwise invasibility plot along its main diagonal $x^{\prime}=x$ [which amounts to reversing the roles of the mutant and the resident and gives the sign plot of $\left.f\left(x, x^{\prime}\right)\right]$ and then superimposing the mirror image on the original. The overlapping gray areas correspond to strategy pairs that form protected dimorphisms.

With small mutations, $x$ and $x^{\prime}$ are never far apart, so that only a narrow band along the main diagonal $x^{\prime}=x$ is of immediate interest. The main diagonal itself is always a borderline between "invasion" (gray) and "noninvasion" (white) areas, because residents are selectively neutral among themselves, and therefore $f(x)=0$ for all $x$. In Figure 2a, resident populations with a trait value less than $x^{*}$ can always be invaded by mutants with slightly larger trait values. Coexistence is not possible, because away from $x^{*}$ any combination of mutant and resident strategies near the main diagonal lies within the white area of Figure 2b. Thus, starting with a trait value left of $x^{*}$, the population evolves to the right through a series of successive substitutions. By the same argument, it follows that a population starting on the right of $x^{*}$ evolves to the left. Eventually, the population approaches $x^{*}$, where directional selection ceases. Trait values for which there is no directional selection are called evolutionarily singular strategies (Metz et al. 1996; Geritz et al. 1998).

The graphic analysis of Figure 2 is sufficient to establish the direction of evolution in the case of monomorphic populations in which a single trait is evolving, but gives no explicit information on the speed of evolution. In Box 2, we outline a quantitative approach that assesses the speed of mutation-limited evolution.

\section{The Emergence of Diversity: Evolutionary Branching}

Although the evolutionarily singular strategy $x^{*}$ in Figure 2a is an attractor of monomorphic directional evolution, it is not evolutionarily stable in the classic sense (Maynard Smith 1982), that is, it is not stable against invading mutants. In fact, mutants both smaller and larger than $x^{*}$ can invade the resident population of $x^{*}$. Unlike in directional evolution, in the neighborhood of $x^{*}$ the 
Box 2 The speed of directional evolution

The speed of mutation-limited evolution is influenced by three factors: how often a new mutation occurs; how large a phenotypic change this causes; and how likely it is that an initially rare mutant invades. If the individual mutational steps are sufficiently small, and thus long-term evolution proceeds by a large number of subsequent invasions and substitutions, the evolutionary process can be approximated by the canonical equation of adaptive dynamics (Dieckmann and Law 1996),

$$
\frac{\mathrm{d} x}{\mathrm{~d} t}=\left.\frac{1}{2} \alpha(x) \mu(x) \hat{N}(x) \sigma_{\mathrm{M}}^{2}(x) \frac{\partial f\left(x^{\prime}, x\right)}{\partial x^{\prime}}\right|_{x^{\prime}=x} .
$$

Here $\mu$ is the probability of a mutation per birth event, and $\hat{N}$ is the equilibrium population size: the product $\mu \hat{N}$ is thus proportional to the number of mutations that occur per unit of time. The variance of the phenotypic effect of a mutation is $\sigma_{\mathrm{M}}^{2}$ (with symmetric unbiased mutations, the expected phenotypic effect is zero and the variance measures the size of "typical" mutations). The probability of invasion consists of three factors. First, during directional evolution, either only mutants with a trait value larger than the resident, or only mutants with a trait value smaller than the resident, can invade (see Figure 2a); in other words, half of the mutants are at a selective disadvantage and doomed to extinction. This leads to the factor $\frac{1}{2}$. Second, even mutants at selective advantage may be lost through demographic stochasticity (genetic drift) in the initial phase of invasion, when they are present in only small numbers. For mutants of small effect, the probability of not being lost is proportional to the selective advantage of the mutant as measured by the fitness gradient $\partial f\left(x^{\prime}, x\right) /\left.\partial x^{\prime}\right|_{x^{\prime}=x}$. Finally, the constant of proportionality $\alpha$ is proportional to the inverse of the variance in offspring number: with the same expected number of offspring, an advantageous mutant is more easily lost through demographic stochasticity if its offspring number is highly variable. The constant $\alpha$ equals 1 for a constant birth-death process in an unstructured population, as considered by Dieckmann and Law (1996).

Other models of adaptive dynamics agree that the change in phenotype is proportional to the fitness gradient, that is

$$
\frac{\mathrm{d} x}{\mathrm{~d} t}=\left.\beta \frac{\partial f\left(x^{\prime}, x\right)}{\partial x^{\prime}}\right|_{x^{\prime}=x}
$$

(e.g., Abrams et al. 1993a; Vincent et al. 1993; Marrow et al. 1996). This equation leads to results similar to those from quantitative genetic models (Taper and Case 1992) and, indeed, can be derived as an approximation to the quantitative genetic iteration (Abrams et al. 1993b). Equations (a) and (b) have a similar form, though the interpretation of their terms is different: in quantitative genetics, $\beta$ is the additive genetic variance and thus measures the standing variation upon which selection operates; it is often assumed to be constant. In the canonical equation, $\beta$ depends on the probability and distribution of new mutations; also, $\beta$ generally depends on the prevalent phenotype $x$, if only through the population size $\hat{N}(x)$. In quantitative genetics, evolutionary change is proportional to the fitness gradient, because stronger selection means faster change in the frequencies of alleles that are present from the onset. In mutation-limited evolution, a higher fitness gradient increases the probability that a favorable mutant escapes extinction by demographic stochasticity.

invasion of a mutant results in coexistence of the resident and mutant strategies (Figure 2b). As the singularity is approached by small but finite mutational steps, the population actually becomes dimorphic as soon as the next mutant enters the area of coexistence (i.e., a little before exactly reaching the singular strategy, Figure $2 b$ ).

To see how evolution proceeds in the now dimorphic population, it is useful to plot the mutant's fitness as a function of the mutant trait value (Figure 3). In the resident population of the singular strategy $x^{*}$, all nearby mutants are able to invade (i.e., they have positive fitness), except for the singular strategy itself, which has zero fitness. The fitness function thus attains a minimum at $x^{*}$ (Figure 3a). In a dimorphic population with two strategies $x_{1}$ and $x_{2}$, both similar to $x^{*}$, the fitness 

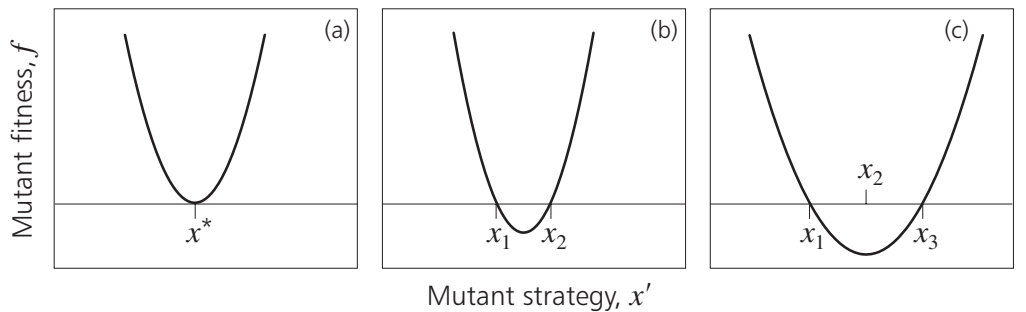

Figure 3 Evolutionary branching and a mutant's fitness as a function of its strategy. (a) Mutant fitness in a monomorphic resident population at a branching point $x^{*}$. (b) Mutant fitness in a dimorphic resident population with strategies $x_{1}$ and $x_{2}$, both similar to the branching point $x^{*}$. Notice that only those mutants outside the interval spanned by $x_{1}$ and $x_{2}$ have a positive fitness and hence can invade. (c) Mutant fitness in a dimorphic resident population with strategies $x_{1}$ and $x_{3}$. The former resident $x_{2}$ now has a negative fitness, and hence is expelled from the population.

function is also similar, but with zeros at $x_{1}$ and $x_{2}$, because residents themselves are selectively neutral (Figure 3b).

According to Figure $3 \mathrm{~b}$, a new mutant that arises in the dimorphic population with strategies $x_{1}$ and $x_{2}$ similar to $x^{*}$ has a positive fitness, and therefore can invade, if and only if it is outside the interval spanned by the two resident trait values. By contrast, mutants between these values have a negative fitness and therefore must die out. A mutant cannot coexist with both former residents, because the parabolically shaped fitness function cannot have three zeros to accommodate three established resident strategies. It follows that the successfully invading mutant will oust the resident that has become the middle strategy (Figure 3c).

Since the initial dimorphic population is formed of the most recent monomorphic resident and its mutant, with small mutations these two strategies are very similar. After the first substitution in the dimorphic population, however, the new resident population consists of two strategies with a wider gap between. Through a series of such invasions and replacements, the two strategies of the dimorphic population undergo divergent coevolution and become phenotypically clearly distinct (see Figure 1).

The process of convergence to a particular trait value in the monomorphic population followed by gradual divergence once the population has become dimorphic is called evolutionary branching. The singularity at which this happens ( $x^{*}$ in Figure 2a) is an evolutionary branching point. In Box 3 we summarize how to recognize branching points by investigating the fitness function $f\left(x^{\prime}, x\right)$.

The evolutionary branching point, though perhaps the most interesting with regard to speciation, is not the only type of singular strategy. In Box 4 we briefly summarize the basic properties of all singularities that occur generically. Throughout this section, we constrain our discussion to single-trait evolution in an initially monomorphic population. A brief summary on how to extend these results to polymorphic populations (including further branching events as in Figure 1) and to multiple-trait evolution is given in Box 5; more details can be found in Metz et al. (1996) and Geritz et al. (1998, 1999), and, concerning directional evolution, in Dieckmann and Law (1996), Matessi and Di Pasquale (1996), Champagnat et al. (2001), and Leimar (2001 and in press).

For the adaptive dynamics framework to be applicable to spatially subdivided populations, sufficient dispersal must occur between subpopulations for the stationary population distribution to be attained on an ecological time scale. Full sympatry is, however, by no means a necessary condition, and the framework has been used to analyze evolution in spatially structured populations as well (e.g., Meszéna et al. 1997; Day 2000; see Box 6).

So far we have considered clonally inherited phenotypes. The very same model can be applied, however, to the evolution of alleles at a single diploid locus in a Mendelian population [Box 7; Kisdi and Geritz 1999; see also Christiansen and Loeschcke (1980) for a related approach] when 
Box 3 How to recognize evolutionary branching points

One can easily search for evolutionary branching points in a model once the mutant fitness function $f\left(x^{\prime}, x\right)$ has been determined. If $f\left(x^{\prime}, x\right)$ is known analytically, then the following criteria must be satisfied by an evolutionary branching point $x^{*}$ (Geritz et al. 1998):

1. $x^{*}$ must be an evolutionary singularity, i.e., the fitness gradient vanishes at $x^{*}$,

$$
\left.\frac{\partial f\left(x^{\prime}, x\right)}{\partial x^{\prime}}\right|_{x^{\prime}=x=x^{*}}=0 .
$$

2. $x^{*}$ must be an attractor of directional evolution (Eshel 1983),

$$
\frac{\partial^{2} f\left(x^{\prime}, x\right)}{\partial x \partial x^{\prime}}+\left.\frac{\partial^{2} f\left(x^{\prime}, x\right)}{\partial x^{\prime 2}}\right|_{x^{\prime}=x=x^{*}}<0 .
$$

3. In the neighborhood of $x^{*}$, similar strategies must be able to form protected dimorphisms (Geritz et al. 1998),

$$
\frac{\partial^{2} f\left(x^{\prime}, x\right)}{\partial x^{2}}+\left.\frac{\partial^{2} f\left(x^{\prime}, x\right)}{\partial x^{\prime 2}}\right|_{x^{\prime}=x=x^{*}}>0 .
$$

4. $x^{*}$ must lack evolutionary stability (Maynard Smith 1982), which ensures disruptive selection at $x^{*}$ (Geritz et al. 1998),

$$
\left.\frac{\partial^{2} f\left(x^{\prime}, x\right)}{\partial x^{\prime 2}}\right|_{x^{\prime}=x=x^{*}}>0 \text {. }
$$

As can be verified by inspection of all the generic singularities (see Box 4), the second-order criteria (2)-(4) are not independent for the case of a single trait and an initially monomorphic resident population; instead, criteria (2) and (4) are sufficient to ensure (3) as well. This is, however, not true for multidimensional strategies or for coevolving populations (Geritz et al. 1998). These criteria are thus best remembered separately.

Alternatively, a graphic analysis can be performed using a pairwise invasibility plot (Figure 2a). Although drawing the pairwise invasibility plot is practical only for the case of single traits and monomorphic populations, it is often used when the invasion fitness cannot be determined analytically. In a pairwise invasibility plot, the evolutionary branching point is recognized by the following pattern:

- The branching point is at a point of intersection between the main diagonal and another border line between positive and negative mutant fitness.

- The fitness of mutants is positive immediately above the main diagonal to the left of the branching point and below the main diagonal to its right.

- Potentially coexisting strategies lie in the neighborhood of the branching point (this can be checked on a plot similar to Figure 2b, but, as highlighted above, in the simple case for which pairwise invasibility plots are useful, this criterion does not have to be checked separately).

- Looking along a vertical line through the branching point, the mutants immediately above and below are able to invade.

assuming that a continuum of allele types is possible, and that the mutant allele codes for a phenotype similar to that of the parent allele. Evolutionary branching in alleles then occurs similarly to clonal phenotypes and produces two distinct allele types that may continue to segregate within the species. Since intermediate heterozygotes are at a disadvantage under disruptive selection, selection occurs for dominance and for assortative mating (Udovic 1980; Wilson and Turelli 1986; Van Dooren 1999; Geritz and Kisdi 2000). 
Box 4 Types of evolutionary singularities

Eight types of evolutionary singularities occur generically in single-trait evolution of monomorphic populations, as in the figure below (Geritz et al. 1998). As in Figure 2a, gray areas indicate combinations of mutant strategies and resident strategies for which the mutant's fitness is positive.

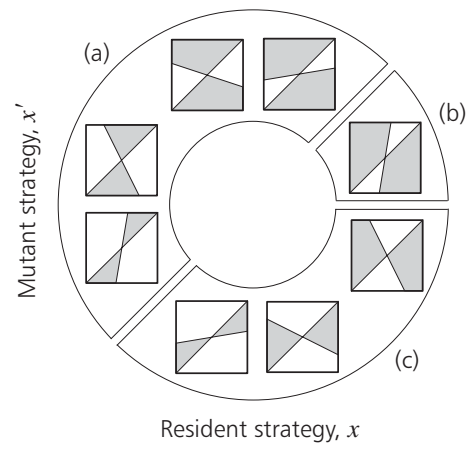

These types can be classified into three major groups:

- Evolutionary repellors, (a) in the figure above. Directional evolution leads away from this type of singularity, and therefore these types do not play a role as evolutionary outcomes. If the population has several singular strategies, then a repellor separates the basins of attraction of adjacent singularities.

- Evolutionary branching points, $(b)$ in the figure above. This type of singularity is an attractor of directional evolution, but it lacks evolutionary stability and therefore evolution cannot stop here. Invading mutants give rise to a protected dimorphism in which the constituent strategies are under disruptive selection and diverge away from each other. Evolution can enter a higher level of polymorphism by small mutational steps only via evolutionary branching.

- Evolutionarily stable attractors, (c) in the figure above. Singularities of this type are attractors of directional evolution and, moreover, once established the population cannot be invaded by any nearby strategy. Such strategies are also called continuously stable strategies (Eshel 1983). Coexistence of strategies may be possible, but coexisting strategies undergo convergent rather than divergent coevolution such that eventually the dimorphism disappears. Evolutionarily stable attractors act as final stops of evolution.

\section{Evolutionary Branching and Speciation}

The phenomenon of evolutionary branching in clonal models may appear very suggestive of speciation. First, there is directional evolution toward a well-defined trait value, the evolutionary branching point. As evolution reaches the branching point, selection turns disruptive. The population necessarily becomes dimorphic in the neighborhood of the branching point, and disruptive selection causes divergent coevolution in the two coexisting lineages. The resultant evolutionary pattern is of a branching evolutionary tree, with phenotypically distinct lineages that develop gradually by small evolutionary steps (Figure 1).

Naturally, clonal models of adaptive dynamics are unable to account for the genetic details of speciation, in particular how reproductive isolation might develop between the emerging branches (see Dieckmann and Doebeli 1999; Drossel and McKane 2000; Geritz and Kisdi 2000; Matessi et al. 2001; Meszéna and Christiansen, unpublished; see Chapter 5 in Dieckmann et al. 2004). What evolutionary branching does imply is that there is evolution toward disruptive selection and, at the same time, toward polymorphism in the ecological model in which branching is found. These are the ecological prerequisites for speciation and set the selective environment for the evolution of reproductive isolation. Evolutionary branching thus indicates that the ecological system under study is prone to speciation. 
Box 5 Polymorphic and multidimensional evolution

If the theory of adaptive dynamics were only applicable to the caricature of one-dimensional trait spaces or monomorphic populations, it would be of very limited utility. Below we therefore describe how this framework can be extended.

We start by considering polymorphic populations. By assuming mutation-limited evolution we can ignore the possibility of simultaneous mutations that occur in different resident strategies. Two strategies $x_{1}$ and $x_{2}$ can coexist as a protected dimorphism if both $f\left(x_{2}, x_{1}\right)$ and $f\left(x_{1}, x_{2}\right)$ are positive (i.e., when both can invade into a population of the other). For each pair of resident strategies $x_{1}$ and $x_{2}$ we can construct a pairwise invasibility plot for $x_{1}$ while keeping $x_{2}$ fixed, and a pairwise invasibility plot for $x_{2}$ while keeping $x_{1}$ fixed. From this we can see which mutants of $x_{1}$ or of $x_{2}$ could invade the present resident population and which could not (i.e., in what direction $x_{1}$ and $x_{2}$ will evolve by small mutational steps).

In the example shown in the figure below, the arrows indicate the directions of evolutionary change in $x_{1}$ and in $x_{2}$. On the lines that separate regions with different evolutionary directions, selection in one of the two resident strategies is no longer directional: each point on such a line is a singular strategy for the corresponding resident, if the other resident is kept fixed. The points of these lines, therefore, can be classified similarly to the monomorphic singularities in Box 4. Within the regions of coexistence in the figure below continuous lines indicate evolutionary stability and dashed lines the lack thereof. At the intersection point of two such lines, directional evolution ceases for both residents. Such a strategy combination is called an evolutionarily singular dimorphism. This dimorphism is evolutionarily stable if neither mutants of $x_{1}$ nor mutants of $x_{2}$ can invade (i.e., if both $x_{1}$ and $x_{2}$ are evolutionarily stable); in the figure this is the case. For a singular dimorphism to be evolutionarily attracting it is neither necessary nor sufficient that both strategies are attracting if the other resident is kept fixed at its present value (Matessi and Di Pasquale 1996; Marrow et al. 1996). With small evolutionary steps, we can approximate the evolutionary trajectories by utilizing the canonical equation (see Box 2) simultaneously for both coevolving strategies. Stable equilibria of the canonical equation then correspond to evolutionarily attracting singular dimorphisms. If such a dimorphism is evolutionarily stable, it represents a final stop of dimorphic evolution. However, if one of the resident strategies at the singularity is not evolutionarily stable and, moreover, if this resident can coexist with nearby mutants of itself, the population undergoes a secondary branching event, which leads to a trimorphic resident population (Metz et al. 1996; Geritz et al. 1998). An example of such a process is shown in Figure 1.

continued

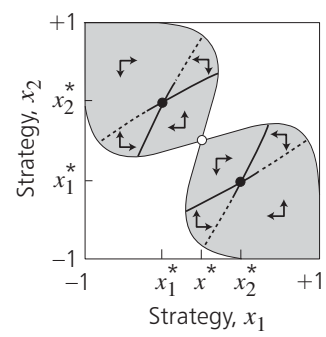

Adaptive dynamics in a dimorphic population for the model described in Box 1 with $r=1, K(x)=\left(1-x^{2}\right)_{+}$, and $\sigma_{a}=0.5$. Gray areas indicate strategy pairs $\left(x_{1}, x_{2}\right)$ that can coexist as a protected dimorphism. Lines inside the gray areas separate regions with different evolutionary directions for the two resident strategies, as illustrated by arrows. On the steeper line, which separates strategy pairs evolving either toward the left or toward the right, directional evolution in $x_{1}$ ceases. Likewise, on the shallower line, which separates strategy pairs evolving upward from those evolving downward, directional evolution in $x_{2}$ ceases. Continuous lines indicate that the corresponding strategy is evolutionarily stable if evolution in the other strategy is arrested. After branching at the branching point $x^{*}$ (open circle), the population evolves into the gray area toward the evolutionarily stable dimorphism $\left(x_{1}^{*}, x_{2}^{*}\right.$ ) (filled circles), where directional evolution ceases in both strategies and both strategies also possess evolutionary stability. 


\section{Box 5 continued}

Next we consider the adaptive dynamics framework in the context of multidimensional strategies. In natural environments, strategies are typically characterized by several traits that jointly influence fitness and that may be genetically correlated.

Though much of the basic framework can be generalized to multidimensional strategies, these also pose special difficulties. For example, unlike in the case of scalar strategies, a mutant that invades a monomorphic resident population may coexist with the former resident also away from any evolutionary singularity. This coexistence, however, is confined to a restricted set of mutants, such that its volume vanishes for small mutational steps proportionally to the square of the average size of mutations. With this caveat, directional evolution of two traits in a monomorphic population can be depicted graphically in a similar way to coevolving strategies. There are two important differences, however. First, the axes of the figure on the previous page no longer represent different residents, but instead describe different phenotypic components of the same resident phenotype. Second, if the traits are genetically correlated such that a single mutation can affect both traits at the same time, then the evolutionary steps are not constrained to being either horizontal or vertical. Instead, evolutionary steps are possible in any direction within an angle of plus or minus 90 degrees from the selection gradient vector $\partial f\left(x^{\prime}, x\right) /\left.\partial x^{\prime}\right|_{x^{\prime}=x}$.

For small mutational steps, the evolutionary trajectory can be approximated by a multidimensional equivalent of the canonical equation (Dieckmann and Law 1996; see Box 2), where $\mathrm{d} x / \mathrm{d} t$ and $\partial f\left(x^{\prime}, x\right) /\left.\partial x^{\prime}\right|_{x^{\prime}=x}$ are vectors, and the mutational variance is replaced by the mutation variancecovariance matrix $C(x)$ (the diagonal elements of this matrix contain the trait-wise mutational variances and the off-diagonal elements represent the covariances between mutational changes in two different traits that may result from pleiotropy). With large covariances, it is possible that a trait changes "maladaptively", that is, the direction of the net change is opposite to the direct selection on the trait given by the corresponding component of the fitness gradient (see also Lande 1979b).

An evolutionarily singular strategy $x^{*}$, in which all components of the fitness gradient are zero, is evolutionarily stable if it is, as a function of the traits of the mutant strategy $x^{\prime}$, a multidimensional maximum of the invasion fitness $f\left(x^{\prime}, x^{*}\right)$. If such a singularity lacks evolutionary stability, evolutionary branching may occur.

Speciation by disruptive selection has previously been considered problematic, because disruptive selection does not appear to be likely to occur for a long time and does not appear to be compatible with the coexistence of different types (either different alleles or different clonal types or species). For disruptive selection to occur, the population must be at the bottom of a fitness valley (similar to Figure 3). In simple, frequency-independent models of selection, the population "climbs" toward the nearest peak of the adaptive landscape (Wright 1931; Lande 1976). The fitness valleys are thus evolutionary repellors: the population is unlikely to experience disruptive selection, except possibly for a brief exposure before it evolves away from the bottom of the valley.

As pointed out by Christiansen (1991) and Abrams et al. (1993a), evolution by frequencydependent selection often leads to fitness minima. Even though in each generation the population evolves "upward" on the fitness landscape, the landscape itself changes such that the population eventually reaches the bottom of a valley. This is what happens during directional evolution toward an evolutionary branching point.

Disruptive selection has also been thought incompatible with the maintenance of genetic variability (e.g., Ridley 1993). In simple one-locus models disruptive selection amounts to heterozygote inferiority, which, in the absence of frequency dependence, leads to the loss of one allele. This is not so under frequency dependence (Pimm 1979): at the branching point, the heterozygote is inferior only when both alleles are sufficiently common. Should one of the alleles become rare, the frequency-dependent fitness of the heterozygote increases such that it is no longer at a disadvantage, and therefore the frequency of the rare allele increases again. 
Box 6 The geography of speciation

Evolutionary branching in a spatially subdivided population based on a simple model by Meszéna et al. (1997) is illustrated here. Two habitats coupled by migration are considered. Within each habitat, the population follows logistic growth, in which the intrinsic growth rate is a Gaussian function of strategy, with different optima in the two habitats. The model is symmetric, so that the "generalist" strategy, which is exactly halfway between the two habitat-specific optima, is always an evolutionarily singular strategy. Depending on the magnitude of the difference $\Delta$ between the local optima relative to the width of the Gaussian curve and on the migration rate $m$, this central singularity may either be an evolutionarily stable strategy, a branching point, or a repellor [(a) in the figure below; see also Box 4].

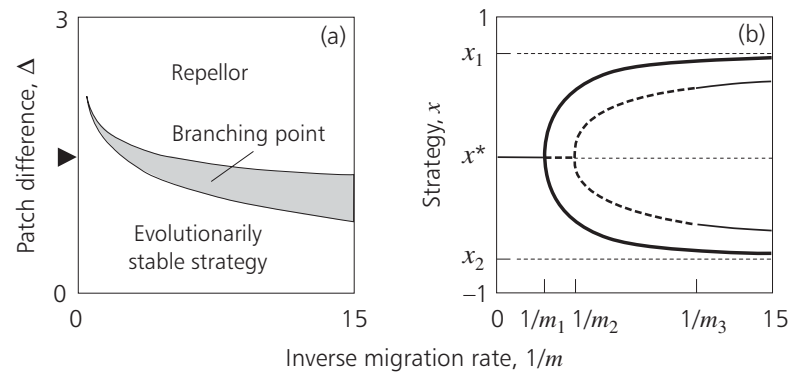

Evolutionary properties of singularities in a two-patch model with local adaptation and migration. (a) A generalist strategy that exploits both patches is an evolutionary repellor, a branching point, or an evolutionarily stable attractor, depending on the difference $\Delta$ between the patch-specific optimal strategies and the migration rate $m$, as indicated by the three different parameter regions. (b) Evolutionary singularities as a function of inverse migration rate. The difference between the patch-specific optimal strategies was fixed at $\Delta=1.5$. For comparison, the two thin dotted horizontal lines at $x_{1}$ and $x_{2}$ denote the local within-patch optimal strategies $(x= \pm \Delta / 2)$. Monomorphic singular strategies are drawn with lines of intermediate thickness, of which the continuous lines correspond to evolutionarily stable attractors, the dashed lines to branching points, and the dotted line to an evolutionary repellor. The monomorphic generalist strategy is indicated by $x^{*}$. Along a crosssection at $\Delta=1.5$ in (a), indicated by an arrow head, the generalist strategy changes with increasing $1 / \mathrm{m}$ from an evolutionarily stable attractor into a branching point and then into an evolutionary repellor. The two branches of the bold line indicate the strategies of the evolutionarily stable dimorphism. Source: Meszéna et al. (1997).

There are four possible evolutionary scenarios [(b) in the figure above]: at high levels of migration (inverse migration rate smaller than $1 / m_{1}$ ), the population effectively experiences a homogeneous environment in which the generalist strategy is evolutionarily stable and branching is not possible. With a somewhat lower migration rate (inverse migration rate between $1 / m_{1}$ and $1 / m_{2}$ ), the generalist is at an evolutionary branching point, and the population evolves to a dimorphism that consists of two habitat specialists. Decreasing migration further (inverse migration rate between $1 / m_{2}$ and $\left.1 / m_{3}\right)$, the generalist becomes an evolutionary repellor, but there are two additional monomorphic singularities, one on each side of the generalist, both of which are branching points. Finally, in case of a very low migration rate (inverse migration rate greater than $1 / m_{3}$ ), these two monomorphic attractors are evolutionarily stable and branching does not occur, even though there also exists an evolutionarily stable dimorphism of habitat specialists. (A similar sequence of transitions can be observed if, instead of decreasing the migration rate, the difference between the habitats is increased.) Evolutionary branching is also possible if the environment forms a gradient instead of discrete habitats, provided there are sufficiently different environments along the gradient and mobility is not too high (Mizera and Meszéna 2003; Chapter 7 in Dieckmann et al. 2004). 
Box 7 Adaptive dynamics of alleles and stable genetic polymorphisms

As an example for the adaptive dynamics of alleles, consider the classic soft-selection model of Levene (1953; see Box 3.1 in Dieckmann et al. 2004). We assume that not just two alleles may segregate (A and a, with fixed selection coefficients $s_{1}$ and $s_{2}$ ), as in the classic models, but instead that many different alleles may arise by mutations and that they determine a continuous phenotype in an additive way (i.e., if the phenotypes of AA and aa are, respectively, $x_{\mathrm{A}}$ and $x_{\mathrm{a}}$, then the heterozygote phenotype is $\left.\left(x_{\mathrm{A}}+x_{\mathrm{a}}\right) / 2\right)$. Within each habitat, local fitness is a function of the phenotype: $\varphi_{i}(x)$ in habitat $i$. For example, in the first habitat local fitness values are $W_{\mathrm{AA}}=\varphi_{1}\left(x_{\mathrm{A}}\right), W_{\mathrm{Aa}}=\varphi_{1}\left(\left(x_{\mathrm{A}}+x_{\mathrm{a}}\right) / 2\right)$, and $W_{\mathrm{aa}}=\varphi_{1}\left(x_{\mathrm{a}}\right)$. For any two alleles $\mathrm{A}$ and a, drawn from the assumed continuum, the dynamics and equilibrium of allele frequencies can be obtained as described in Box 3.1 in Dieckmann et al. 2004. In particular, the frequency of a rare mutant allele a increases in a population monomorphic for allele $\mathrm{A}$ at a per-generation rate of $k_{1} \varphi_{1}\left(\left(x_{\mathrm{A}}+x_{\mathrm{a}}\right) / 2\right) / \varphi_{1}\left(x_{\mathrm{A}}\right)+k_{2} \varphi_{2}\left(\left(x_{\mathrm{A}}+x_{\mathrm{a}}\right) / 2\right) / \varphi_{2}\left(x_{\mathrm{A}}\right)$, where $k_{i}$ is the relative size of habitat $i$, with $k_{1}+k_{2}=1$. If this expression is greater than 1 [or, equivalently, if its logarithm, $f\left(x_{\mathrm{a}}, x_{\mathrm{A}}\right)$ in the notation of the main text, is positive], then the mutant allele can invade.

Assuming that mutations only result in small phenotypic change ( $x_{\mathrm{a}}$ is near $\left.x_{\mathrm{A}}\right)$, we can apply the adaptive dynamics framework to the evolution of alleles (Geritz et al. 1998). Invasion by a mutant allele usually leads to substitution (i.e., the new allele replaces the former allele, just as in clonal adaptive dynamics). The ensuing directional evolution, however, leads to singular alleles in which protected polymorphisms become possible. Evolutionary branching of alleles means that the homozygote phenotypes diverge from each other, and results in a genetic polymorphism of distinctly different alleles that segregate in a randomly mating population (Kisdi and Geritz 1999).

Assuming a more flexible genetic variation sheds new light on the old question of whether stable genetic polymorphisms are sufficiently robust to serve as a basis for sympatric speciation. Recall from Box 3.1 in Dieckmann et al. 2004 that if selection coefficients $s_{i}$ are small, then polymorphism is possible only in a very narrow range of parameters (the parameter region that allows for polymorphism actually has a cusp at $s_{1}=s_{2}=0$ ). Given two arbitrary alleles A and a, and therefore given selection coefficients $s_{1}$ and $s_{2}$, polymorphism results only if the environmental parameters, in this case the relative habitat sizes $k_{1}$ and $k_{2}=1-k_{1}$, are fine-tuned. This means that a polymorphism of two particular alleles is not robust under weak selection (Maynard Smith 1966; Hoekstra et al. 1985), and this property appeared a significant obstacle to sympatric speciation.

By contrast, the assumption of more flexible genetic variation (a potential continuum of alleles rather than only two alleles) considerably facilitates the evolution of stable genetic polymorphisms. Here we focus on polymorphisms of similar alleles (which may arise by a single mutation at the onset of evolutionary branching); this immediately implies that the selection coefficients are small and that the two alleles cannot form a polymorphism without fine-tuning of the environmental parameters. With many potential alleles, however, the requirement of fine-tuning may be turned around: given a certain environment $\left(k_{1}\right.$ and $\left.k_{2}\right)$, polymorphism will result if the alleles are chosen from a narrow range. This narrow range turns out to coincide with the neighborhood of an evolutionarily singular allele. Thus, starting with an arbitrary allele A, population genetics and adaptive dynamics agree in that the invasion of a mutant allele a usually results in substitution rather than polymorphism. Repeated substitutions, however, lead toward a singular allele, in the neighborhood of which stable polymorphisms are possible. In other words, evolution by small mutational steps proceeds exactly toward those exceptional alleles that can form polymorphisms: long-term evolution itself takes care of the necessary fine-tuning (Kisdi and Geritz 1999; see figure below). If the singularity is a branching point, then the population not only becomes genetically polymorphic, but also is subject to disruptive selection, as is necessary for sympatric speciation. Of course, it remains to be seen whether reproductive isolation can evolve [see Chapters 3 and 5 in Dieckmann et al. 2004 and references therein; see also Geritz and Kisdi (2000) for an analysis of the evolution of reproductive isolation through the adaptive dynamics of alleles]. Fine-tuning is necessary not only in multiple-niche polymorphisms [such as Levene's (1953) model and its variants, see Hoekstra et al. (1985)], but also in any generic model in which frequency dependence can maintain protected polymorphisms; long-term evolution then provides the necessary fine-tuning whenever many small mutations incrementally change an evolving trait.

continued 
Box 7 continued
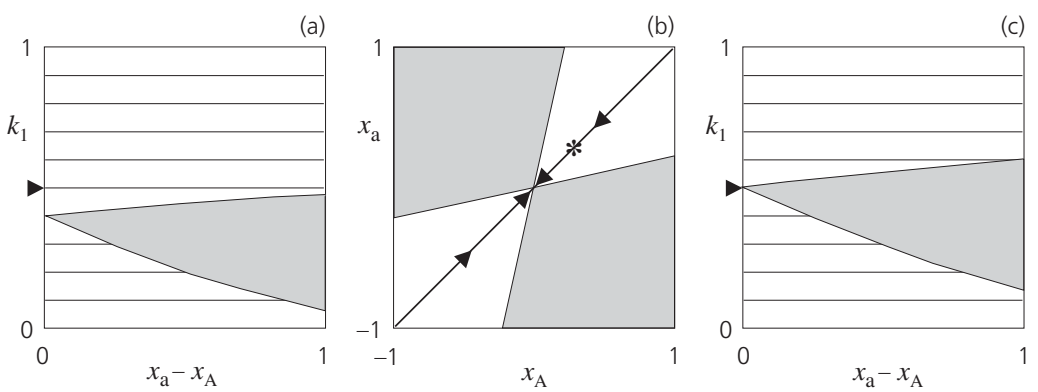

Allele pairs that can form a stable polymorphism in Levene's soft selection model with stabilizing selection within each habitat $\left(\varphi_{i}\right.$ is Gaussian with unit width and the two peaks are located at a distance of $\Delta=3$ ). (a) An arbitrary allele $x_{\mathrm{A}}=0.3 \mathrm{can}$ form a polymorphism with allele $x_{\mathrm{a}}$ within the shaded area. Notice that if $x_{\mathrm{a}}-x_{\mathrm{A}}$ is small (i.e., if the two alleles produce similar phenotypes and hence selection is weak), then polymorphism is possible only in a narrow range of the environmental parameter $k_{1}$ (fine-tuning). In one particular environment $k_{1}=0.5$ (indicated by arrowheads in the left and right panels), the allele $x_{\mathrm{A}}=0.3$ cannot form a polymorphism under weak selection. (b) The set of allele pairs that can form polymorphisms in the particular environment $k_{1}=0.5$. The thick line corresponds to identical alleles $x_{\mathrm{a}}=x_{\mathrm{A}}$; similar alleles with small difference $x_{\mathrm{a}}-x_{\mathrm{A}}$ thus lie in the neighborhood of the thick line. Again, the allele $x_{\mathrm{A}}=0.3$ (denoted by an asterisk) cannot form a polymorphism with alleles similar to itself in this particular environment. Allele substitutions in the monomorphic population, however, lead to the evolutionary singular allele $x^{*}=0$, where similar alleles are able to form polymorphisms in this particular environment. (c) With $x_{\mathrm{A}}=x^{*}$, the narrow range of $k_{1}$ that permits polymorphism with small $x_{\mathrm{a}}-x_{\mathrm{A}}$ shifts to the actual value of the environmental parameter, $k_{1}=0.5$. Note that $x^{*}$ depends on the actual value of the environmental parameter; it happens to be the central strategy only for the particular choice $k_{1}=0.5$.

Except for asexual species, evolutionary branching corresponds to speciation only if reproductive isolation emerges between the nascent branches. There are many ways by which reproductive isolation could, in principle, evolve during evolutionary branching (see Chapter 3 in Dieckmann et al. 2004). Assortative mating based on the same ecological trait that is under disruptive selection automatically leads to reproductive isolation as the ecological trait diverges (Drossel and McKane 2000), and this possibility appears to be widespread in nature (e.g., Chown and Smith 1993; Wood and Foote 1996; Macnair and Gardner 1998; Nagel and Schluter 1998; Grant et al. 2000). For example, in the apple maggot fly Rhagoletis pomonella, there is disruptive selection on eclosion time; different timing of reproduction helps to prevent hybridization between the host races (Feder 1998; see Chapter 11 in Dieckmann et al. 2004). If differences in the ecological trait are associated with different habitats, as is the case for the apple maggot fly, then reduced migration, habitat fidelity, or habitat choice ensures assortative mating (Balkau and Feldman 1973; Diehl and Bush 1989; Kawecki 1996, 1997). Assortative mating based on a neutral "marker" trait (e.g., different flower colors that attract different pollinators) can lead to reproductive isolation between the emerging branches only if an association (linkage disequilibrium) is established between the ecological trait and the marker. This is considered to be difficult because of recombination (Felsenstein 1981; but see Dieckmann and Doebeli 1999; Chapter 5 in Dieckmann et al. 2004), and possible only if there is strong assortativeness, strong selection on the ecological trait, or low recombination (Udovic 1980; Kondrashov and Kondrashov 1999; Geritz and Kisdi 2000).

The degree of assortativeness in any mate choice system may be sufficiently high at the onset, or else it may increase evolutionarily while the population is at a branching point. Since disruptive selection acts against intermediate phenotypes, assortative mating between phenotypically similar individuals is selectively favored at the branching point. Adaptive increase of assortativeness may be suspected if mating is more discriminative in sympatry (Coyne and Orr 1989, 1997; Noor 
1995; Sætre et al. 1997) or if some unusual preference appears as a derived character (Rundle and Schluter 1998). In models, increased assortativeness readily evolves if it amounts to the substitution of the same allele in the entire population [a "one-allele mechanism" in the sense of Felsenstein (1981)], such as an allele for increased "choosiness" when selecting mates based on the ecological trait (Dieckmann and Doebeli 1999; Chapter 5 in Dieckmann et al. 2004; but see Matessi et al. 2001; Meszéna and Christiansen, unpublished) or on an allele for reduced migration (Balkau and Feldman 1973). By contrast, two-allele mechanisms depend on the replacement of different alleles in the two branches and thus involve the emergence of linkage disequilibria, a process counteracted by recombination (Felsenstein 1981). Yet such mechanisms have been shown to evolve under certain conditions: when different alleles in the two branches code for different habitat preferences, the process is aided by spatial segregation (Diehl and Bush 1989; Kawecki 1996, 1997), and when the different alleles code for ecologically neutral marker traits, linkage disequilibria can arise from the deterministic amplification of genetic drift in finite populations (Dieckmann and Doebeli 1999; see Chapter 5 in Dieckmann et al. 2004). Partial reproductive isolation by one mechanism facilitates the evolution of other isolating mechanisms (Johnson et al. 1996b), whereby the remaining gene flow is further reduced and the divergent subpopulations attain species rank.

Alternatively, reproductive isolation may arise for reasons independent of disruptive natural selection on the ecological trait. Such mechanisms include sexual selection (Turner and Burrows 1995; Payne and Krakauer 1997; Seehausen et al. 1997; Higashi et al. 1999) and the evolution of gamete-recognition systems (Palumbi 1992). If the emergent species experience directional or stabilizing natural selection, they remain ecologically undifferentiated and hence they are unlikely to coexist for a long time. Evolutionary branching, however, can latch on such that the two species evolve into two branches, which ensures the ecological differentiation necessary for long-term coexistence (Galis and Metz 1998; Van Doorn and Weissing 2001). Once reproductive isolation has been established between the branches in any way, further coevolution of the species proceeds as in the clonal model of adaptive dynamics.

\section{Adaptive Dynamics: Alternative Approaches}

In this chapter, we concentrate on the adaptive dynamics framework developed by Metz et al. (1996) and Geritz et al. (1997, 1998). This is by no means the only approach to adaptive dynamics [see Abrams (2001a) for a review]. We focus on this particular approach because the concept of evolutionary branching may help in the study of nonallopatric speciation. Alternative approaches consider the number of species fixed [and hence do not consider speciation at all; see Abrams (2001a) for references to many examples], or assume invasions of new species from outside the system [the invading species in such cases may be considerably different from the members of the present community and its phenotype is more or less arbitrary; e.g., Taper and Case (1992)], or establish that polymorphism will occur at fitness minima, but do not investigate the subsequent coevolution of the constituent strategies (e.g., Brown and Pavlovic 1992). An important exception is the work of Eshel et al. (1997), which paralleled some results of Metz et al. (1996) and Geritz et al. (1997, 1998). A recent paper by Cohen et al. (1999) gives similar results to those presented in this chapter. This approach uses differential equations to describe the convergence to the branching point in a monomorphic population and divergence in a dimorphic population; to incorporate the transition from monomorphism to dimorphism, the model has to be modified by adding a new equation at the branching point (see, however, Abrams 2001b). Most models of adaptive dynamics agree on the basic form of the equation that describes within-species phenotypic change over time [see Box 2, Equation (b)]. 
In the present framework, analytical tractability comes at the cost of assuming mutation-limited evolution, that is, assuming mutations that occur infrequently and, if successful, sweep through the population before the next mutant comes along. Simulations of the evolutionary process (similar to that shown in Figure 1, but with variable size and frequency of mutations) demonstrate that the qualitative patterns of monomorphic evolution and evolutionary branching are robust with respect to relaxing this assumption. With a higher frequency of mutations, the next mutant arises before the previous successful mutant has become fixed, and therefore there is always some variation in the population. The results are robust with respect to this variation because the environment, $E$ in Equations (1a) and (1b), generated by a cluster of similar strategies is virtually the same if the cluster is replaced by a single resident. Therefore, there is no qualitative difference in terms of which strategies can invade.

\section{Concluding Comments}

Recent empirical research has highlighted the significance of adaptive speciation (e.g., Schluter and Nagel 1995; Schluter 1996a; Losos et al. 1998; Schneider et al. 1999; Schilthuizen 2000); in many instances, natural selection plays a decisive role in species diversification. It is a challenge for evolutionary theory to construct an appropriate theoretical framework for adaptive speciation. Adaptive dynamics provides one facet as it identifies speciation-prone ecological conditions, in which selection favors diversification with ecological contact.

Classic speciation models (e.g., Udovic 1980; Felsenstein 1981; Kondrashov and Kondrashov 1999) emphasize the population genetics of reproductive isolation, and merely assume some disruptive selection, either as an arbitrary external force or by incorporating only the simplest ecology [very often a version of Levene's (1953) model with two habitats; see Chapter 3 in Dieckmann et al. 2004]. By contrast, adaptive dynamics focuses on the ecological side of speciation. It offers a theoretical framework for the investigation in complex ecological scenarios as to whether, and under which conditions, speciation can be expected. Beyond the prediction of a certain speciation event, adaptive dynamics can analyze various patterns in the development of species diversity (see Box 8).

On a paleontological time scale, evolution driven by directional selection and, presumably, adaptive speciation is very fast (McCune and Lovejoy 1998; Hendry and Kinnison 1999). It is thus tempting to think of the paleontological record as a series of evolutionarily stable communities, the changes being brought about by some physical change in the environment (Rand and Wilson 1993). The emerging bifurcation theory of adaptive dynamics (Geritz et al. 1999; Jacobs, et al. unpublished; see Box 6 for an example) is capable of studying the properties of evolutionarily stable communities as a function of environmental parameters.

Evolutionary branching has been found in many diverse ecological models including, for example, resource competition (Doebeli 1996a; Metz et al. 1996; Day 2000), interference competition (Geritz et al. 1999; Jansen and Mulder 1999; Kisdi 1999), predator-prey systems (Van der Laan and Hogeweg 1995; Doebeli and Dieckmann 2000), spatially structured populations and metapopulations (Doebeli and Ruxton 1997; Meszéna et al. 1997; Kisdi and Geritz 1999; Parvinen 1999; Mathias et al. 2001; Mathias and Kisdi 2002; Mizera and Meszéna 2003), host-parasite systems (Boots and Haraguchi 1999; Koella and Doebeli 1999), mutualistic interactions (Doebeli and Dieckmann 2000; Law et al. 2001), mating systems (Metz et al. 1992; Cheptou and Mathias 2001; de Jong and Geritz 2001; Maire et al. 2001), prebiotic replicators (Meszéna and Szathmáry 2001), and many more. The evolutionary attractors that correspond to fitness minima found, for example, by Christiansen and Loeschcke (1980), Christiansen (1991), Cohen and Levin (1991), Ludwig and Levin (1991), Brown and Pavlovic (1992), Brown and Vincent (1992), Abrams et al. (1993a), Vincent et al. (1993), Doebeli (1996b), and Law et al. (1997) are all evolutionary branching points. 
Box 8 Pattern predictions

In this box, we collect predictions about macroevolutionary patterns derived from adaptive dynamics. No claim is intended that those predictions are all equally hard, or that they cannot be derived through different arguments.

First assume that the external environment exhibits no changes on the evolutionary time scale. [Note that fluctuations on the ecological time scale, like weather changes, are incorporated in the invasion fitness; see Metz et al. (1992) and Section 2 of this chapter.] Adaptive dynamics theory then predicts that

- Speciation only occurs at specific, and in principle predictable, trait values; here these are called evolutionary branching points.

- The ensuing gradual phenotypic differentiation is initially slow compared to the preceding and ensuing periods of directional evolution. Populations sitting near a branching point experience a locally flat fitness landscape, i.e., far weaker selective pressure than during directional selection. Weak selection slows divergence even when assortative mating is readily established. This prediction rests on the assumption that phenotypic variation is narrow compared to the curvature of the fitness function (see Abrams et al. 1993b).

- Speciation typically is splitting into two (i.e., not three or more). As argued in the main text, for one-dimensional phenotypes the geometry of the fitness landscape near branching points precludes the coexistence of more than two types. With multi-dimensional traits, three or more coexisting types can arise in but a few mutational steps (Metz et al. 1996). However, we recently showed that the coevolving incipient species generically align in one dominant direction, making the process effectively one-dimensional.

- Starting with low diversity, many models for adaptive speciation show a quick decrease of the rate of speciation over evolutionary time as the community moves toward a joint ESS (see Box 2 for a heuristic explanation). Note, however, that other evolutionary attractors, e.g., evolutionary limit cycles (Dieckmann et al. 1995; van der Laan and Hogeweg 1995; Khibnik and Kondrashov 1997; Kisdi et al. 2001; Dercole et al. 2002; Mathias and Kisdi, in press) are also possible.

If the external environment does change on a time scale comparable to the initial divergence of new species, it generally precludes speciation from taking off (Metz et al. 1996), which can be understood as follows. Speciation only occurs at special trait values. On these points abut cones within which incipient species can, and outside of which they cannot, coexist (see Figure 2 and Box 7). Externally caused environmental changes move those cones around, away from the current pairs of incipient species, and by snuffing out one branch abort the speciation process before its completion.

If the environment changes sufficiently slowly, species keep tracking their adaptive equilibria till the equilibrium structure undergoes a qualitative change. This brings us in the domain of the bifurcation theory of adaptive dynamics (Box 6). Many phenomena seen in the fossil record may be of this type. Two special bifurcations deserve attention. First, if an ESS disappears in a merger with an evolutionary repellor, a punctuation event occurs: the species goes through a fast evolutionary transient toward another evolutionary attractor (Rand and Wilson 1993). Second, if an ESS changes into a branching point, a punctuation event starting with speciation is seen in the fossil record (Metz et al. 1996; Geritz et al. 1999).

An important insight that emerges from adaptive dynamics is that evolution to a fitness minimum occurs frequently in eco-evolutionary models, suggesting that diversification by evolutionary branching may be common in nature. However, there are a number of caveats. Obviously, the accuracy of the prediction hinges on the assumptions made about the (physiological and other) trade-offs and other constraints on the evolving traits, as well as about the ecological interactions and population dynamics as determined by these traits. Most models predict evolution to a fitness minimum only in some parameter regions but not in others; it is usually difficult to make quantitative estimations of critical model parameters. In view of the often ingenious adaptations in nature, 
it seems unlikely that many species are persistently trapped at fitness minima, but empirical difficulties hinder measuring the actual shape of the fitness function. Divergence from the fitness minimum by evolutionary branching in diploid multilocus systems requires reproductive isolation, i.e., speciation (Doebeli 1996a; Dieckmann and Doebeli 1999; Chapter 5 in Dieckmann et al. 2004). If evolution to fitness minima are indeed common, and persistently maladapted species are indeed rare, then adaptive speciation may be prevalent.

\section{References}

Abrams PA (2001a). Modelling the adaptive dynamics of traits involved in inter- and intraspecific interactions: An assessment of three methods. Ecology Letters 4:166-176

Abrams PA (2001b). Adaptive dynamics: Neither F nor G. Evolutionary Ecology Research 3:369_ 373

Abrams PA, Matsuda H \& Harada Y (1993a). Evolutionarily unstable fitness maxima and stable fitness minima of continuous traits. Evolutionary Ecology 7:465-487

Abrams PA, Harada Y \& Matsuda H (1993b). On the relationship between quantitative genetic and ESS models. Evolution 47:982-985

Balkau B \& Feldman MW (1973). Selection for migration modification. Genetics 74:171-174

Boots M \& Haraguchi Y (1999). The evolution of costly resistance in host-parasite systems. The American Naturalist 153:359-370

Brown JS \& Pavlovic NB (1992). Evolution in heterogeneous environments: Effects of migration on habitat specialization. Evolutionary Ecology 6:360-382

Brown JS \& Vincent TL (1992). Organization of predator-prey communities as an evolutionary game. Evolution 46:1269-1283

Caswell H (1989). Matrix Population Models: Construction, Analysis, and Interpretation. Sunderland, MA, USA: Sinauer Associates Inc.

Champagnat N, Ferrière R \& Arous BG (2001). The canonical equation of adaptive dynamics: A mathematical view. Selection 2:73-83

Cheptou PO \& Mathias A (2001). Can varying inbreeding depression select for intermediary selfing rate? The American Naturalist 157:361-373

Chesson P (1994). Multispecies competition in variable environments. Theoretical Population Biology 45:227-276

Chown SL \& Smith VR (1993). Climate change and the short-term impact of feral house mice at the sub-Antarctic Prince Edwards Islands. Oecologia 96:508-516

Christiansen FB (1991). On conditions for evolutionary stability for a continuously varying character. The American Naturalist 138:37-50

Christiansen FB \& Fenchel TM (1977). Theories of Populations in Biological Communities. Berlin, Germany: Springer-Verlag

Christiansen FB \& Loeschcke V (1980). Evolution and intraspecific exploitative competition. I. One locus theory for small additive gene effects. Theoretical Population Biology 18:297313

Cohen D \& Levin SA (1991). Dispersal in patchy environments: The effects of temporal and spatial structure. Theoretical Population Biology 39:63-99

Cohen Y, Vincent TL \& Brown JS (1999). A G-function approach to fitness minima, fitness maxima, evolutionarily stable strategies and adaptive landscapes. Evolutionary Ecology Research 1:923-942

Coyne JA \& Orr HA (1989). Patterns of speciation in Drosophila. Evolution 43:362-381

Coyne JA \& Orr HA (1997). "Patterns of speciation in Drosophila," revisited. Evolution 51:295-303 
Crow JF \& Kimura M (1970). An Introduction to Population Genetics Theory. New York, NY, USA: Harper \& Row

Day T (2000). Competition and the effect of spatial resource heterogeneity on evolutionary diversification. The American Naturalist 155:790-803

De Jong TJ \& Geritz SAH (2001). The role of geitonogamy in the gradual evolution towards dioecy in cosexual plants. Selection 2:133-146

Dercole F, Ferrière R \& Rinaldi S (2002). Ecological bistability and evolutionary reversals under asymmetrical competition. Evolution 56:1081-1090

Dieckmann U, Marrow P \& Law R (1995). Evolutionary cycling in predator-prey interactions: Population dynamics and the Red Queen. Journal of Theoretical Biology 176:91-102

Dieckmann U \& Doebeli M (1999). On the origin of species by sympatric speciation. Nature 400:354-357

Dieckmann U, Doebeli M, Metz JAJ \& Tautz D (2004). Adaptive Speciation. Cambridge, UK: Cambridge University Press.

Dieckmann U \& Law R (1996). The dynamical theory of coevolution: A derivation from stochastic ecological processes. Journal of Mathematical Biology 34:579-612

Diehl SR \& Bush GL (1989). The role of habitat preference in adaptation and speciation. In Speciation and Its Consequences, eds. Otte D \& Endler JA, pp. 345-365. Sunderland, MA, USA: Sinauer Associates Inc.

Doebeli M (1996a). A quantitative genetic model for sympatric speciation. Journal of Evolutionary Biology 9:893-909

Doebeli M (1996b). An explicit genetic model for ecological character displacement. Ecology 77:510-520

Doebeli M \& Dieckmann U (2000). Evolutionary branching and sympatric speciation caused by different types of ecological interactions. The American Naturalist 156:S77-S101

Doebeli M \& Ruxton GD (1997). Evolution of dispersal rates in metapopulation models: Branching and cyclic dynamics in phenotype space. Evolution 51:1730-1741

Drossel B \& McKane A (1999). Ecological character displacement in quantitative genetic models. Journal of Theoretical Biology 196:363-376

Drossel B \& McKane A (2000). Competitive speciation in quantitative genetic models, Journal of Theoretical Biology 204:467-478

Eshel I (1983). Evolutionary and continuous stability. Journal of Theoretical Biology 103:99-111

Eshel I, Motro U \& Sansone E (1997). Continuous stability and evolutionary convergence. Journal of Theoretical Biology 185:333-343

Feder JL (1998). The apple maggot fly, Rhagoletis pomonella: Flies in the face of conventional wisdom about speciation? In Endless Forms: Species and Speciation, eds. Howard DJ \& Berlocher SH, pp. 130-144. Oxford, UK: Oxford University Press

Felsenstein J (1981). Scepticism towards Santa Rosalia, or why are there so few kinds of animals? Evolution 35:124-138

Galis F \& Metz JAJ (1998). Why are there so many cichlid species? Trends in Ecology and Evolution 13:1-2

Geritz SAH \& Kisdi É (2000). Adaptive dynamics in diploid sexual populations and the evolution of reproductive isolation. Proceedings of the Royal Society of London B 267:1671-1678

Geritz SAH, Metz JAJ, Kisdi É \& Meszéna G (1997). Dynamics of adaptation and evolutionary branching. Physical Review Letters 78:2024-2027

Geritz SAH, Kisdi É, Meszéna G \& JAJ Metz (1998). Evolutionarily singular strategies and the adaptive growth and branching of the evolutionary tree. Evolutionary Ecology 12:35-57

Geritz SAH, Van der Meijden E \& Metz JAJ (1999). Evolutionary dynamics of seed size and seedling competitive ability. Theoretical Population Biology 55:324-343 
Goel NS \& Richter-Dyn N (1974). Stochastic Models in Biology. New York, NY, USA: Academic Press

Grant PR, Grant BR \& Petren K (2000). The allopatric phase of speciation: The sharp-beaked ground finch (Geospiza difficilis) on the Galapagos islands. Biological Journal of the Linnean Society 69:287-317

Hendry AP \& Kinnison MT (1999). The pace of modern life: Measuring rates of contemporary microevolution. Evolution 53:1637-1653

Higashi M, Takimoto G \& Yamamura N (1999). Sympatric speciation by sexual selection. Nature 402:523-526

Hoekstra RF, Bijlsma R \& Dolman J (1985). Polymorphism from environmental heterogeneity: Models are only robust if the heterozygote is close in fitness to the favoured homozygote in each environment. Genetical Research (Cambridge) 45:299-314

Hofbauer J \& Sigmund K (1990). Adaptive dynamics and evolutionary stability. Applied Mathematics Letters 3:75-79

Jacobs FJ, Metz JAJ, Geritz SAH \& Meszéna G. Bifurcation analysis for adaptive dynamics based on Lotka-Volterra community dynamics. Unpublished

Jansen VAA \& Mulder GSEE (1999). Evolving biodiversity. Ecology Letters 2:379-386

Johnson PA, Hoppenstaedt FC, Smith JJ \& Bush GL (1996). Conditions for sympatric speciation: A diploid model incorporating habitat fidelity and non-habitat assortative mating. Evolutionary Ecology 10:187-205

Kawecki TJ (1996). Sympatric speciation driven by beneficial mutations. Proceedings of the Royal Society of London B 263:1515-1520

Kawecki TJ (1997). Sympatric speciation driven by deleterious mutations with habitat-specific effects. Evolution 51:1751-1763

Khibnik AI \& Kondrashov AS (1997). Three mechanisms of Red Queen dynamics. Proceedings of the Royal Society of London B 264:1049-1056

Kisdi É (1999). Evolutionary branching under asymmetric competition. Journal of Theoretical Biology 197:149-162

Kisdi É \& Geritz SAH (1999). Adaptive dynamics in allele space: Evolution of genetic polymorphism by small mutations in a heterogeneous environment. Evolution 53:993-1008

Kisdi É \& Meszéna G (1993). Density dependent life history evolution in fluctuating environments. In Adaptation in a Stochastic Environment, eds. Yoshimura J \& Clark C, pp. 26-62, Lecture Notes in Biomathematics Vol. 98. Berlin, Germany: Springer-Verlag

Kisdi É, Jacobs FJA \& Geritz SAH (2001). Red Queen evolution by cycles of evolutionary branching and extinction. Selection 2:161-176

Koella JC \& Doebeli M (1999). Population dynamics and the evolution of virulence in epidemiological models with discrete host generations. Journal of Theoretical Biology 198:461-475

Kondrashov AS \& FA Kondrashov (1999). Interactions among quantitative traits in the course of sympatric speciation. Nature 400:351-354

Lande R (1976). Natural selection and random genetic drift in phenotypic evolution. Evolution 30:314-334

Lande R (1979b). Quantitative genetic analysis of multivariate evolution, applied to brain:body size allometry. Evolution 33:402-416

Law R \& Dieckmann U (1998). Symbiosis through exploitation and the merger of lineages in evolution. Proceedings of the Royal Society of London B 265:1245-1253

Law R, Marrow P \& Dieckmann U (1997). On evolution under asymmetric competition. Evolutionary Ecology 11:485-501

Law R, Bronstein JL \& Ferrière R (2001). On mutualists and exploiters: Plant-insect coevolution in pollinating seed-parasite systems. Journal of Theoretical Biology 212:373-389 
Leimar O (2001). Evolutionary change and Darwinian demons. Selection 2:65-72

Leimar O. Multidimensional convergence stability and the canonical adaptive dynamics. In Elements of Adaptive Dynamics, eds. Dieckmann U \& Metz JAJ. Cambridge, UK Cambridge University Press. In press

Levene H (1953). Genetic equilibrium when more than one ecological niche is available. The American Naturalist 87:331-333

Losos JB, Jackman TR, Larson A, de Queiroz K \& Rodríguez-Schettino L (1998). Contingency and determinism in replicated adaptive radiations of island lizards. Science 279:2115-2118

Ludwig D \& Levin SA (1991). Evolutionary stability of plant communities and the maintenance of multiple dispersal types. Theoretical Population Biology 40:285-307

Macnair MR \& Gardner M (1998). The evolution of edaphic endemics. In Endless Forms: Species and Speciation, eds. Howard DJ \& Berlocher SH, pp. 157-171. Oxford, UK: Oxford University Press

Maire N, Ackermann M \& Doebeli M (2001). Evolutionary branching and the evolution of anisogamy. Selection 2:119-132

Marrow P, Law R \& Cannings C (1992). The coevolution of predator-prey interactions: ESSs and Red Queen dynamics. Proceedings of the Royal Society of London B 250:133-141

Marrow P, Dieckmann U \& Law R (1996). Evolutionary dynamics of predator-prey systems: An ecological perspective. Journal of Mathematical Biology 34:556-578

Matessi C \& Di Pasquale C (1996). Long term evolution of multi-locus traits. Journal of Mathematical Biology 34:613-653

Matessi C, Gimelfarb A \& Gavrilets S (2001). Long term buildup of reproductive isolation promoted by disruptive selection: How far does it go? Selection 2:41-64

Mathias A \& Kisdi É (2002). Adaptive diversification of germination strategies. Proceedings of the Royal Society of London B 269:151-156

Mathias A \& Kisdí É. Evolutionary branching and coexistence of germination strategies. In Elements of Adaptive Dynamics, eds. Dieckmann U \& Metz JAJ. Cambridge, UK: Cambridge University Press. In press

Mathias A, Kisdi É \& Olivieri I (2001). Divergent evolution of dispersal in a heterogeneous landscape. Evolution 55:246-259

Matsuda H (1985). Evolutionarily stable strategies for predator switching. Journal of Theoretical Biology 115:351-366

Maynard Smith J (1966). Sympatric speciation. The American Naturalist 100:637-650

Maynard Smith J (1982). Evolution and the Theory of Games. Cambridge, UK: Cambridge University Press

Maynard Smith J \& Brown RL (1986). Competition and body size. Theoretical Population Biology 30:166-179

Maynard Smith J \& Price GR (1973). The logic of animal conflict, Nature 246:15-18

McCune AR \& Lovejoy NR (1998). The relative rate of sympatric and allopatric speciation in fishes: Tests using DNA sequence divergence between sister species and among clades. In Endless Forms: Species and Speciation, eds. Howard DJ \& Berlocher SH, pp. 172-185. Oxford, UK: Oxford University Press

Meszéna G \& Christiansen FB. On the adaptive emergence of reproductive isolation. Unpublished

Meszéna G \& Szathmáry E (2001). Adaptive dynamics of parabolic replicators. Selection 2:147159

Meszéna G, Czibula I \& Geritz SAH (1997). Adaptive dynamics in a 2-patch environment: A toy model for allopatric and parapatric speciation. Journal of Biological Systems 5:265-284

Metz JAJ, Nisbet RM \& Geritz SAH (1992). How should we define "fitness" for general ecological scenarios? Trends in Ecology and Evolution 7:198-202 
Metz JAJ, Geritz SAH, Meszéna G, Jacobs FJA \& Van Heerwaarden JS (1996). Adaptive dynamics, a geometrical study of the consequences of nearly faithful reproduction. In Stochastic and Spatial Structures of Dynamical Systems, eds. Van Strien SJ \& Verduyn Lunel SM, pp. 183-231. Dordrecht, Netherlands: North Holland

Mizera F \& Meszéna G (2003). Spatial niche packing, character displacement and adaptive speciation along an environmental gradient. Evolutionary Ecology Research 5:363-382

Nagel L \& Schluter D (1998). Body size, natural selection, and speciation in sticklebacks. Evolution 52:209-218

Noor MA (1995). Speciation driven by natural selection in Drosophila. Nature 375:674-675

Nowak M (1990). An evolutionarily stable strategy may be inaccessible. Journal of Theoretical Biology 142:237-241

Palumbi SR (1992). Marine speciation on a small planet. Trends in Ecology and Evolution 7:114118

Parvinen K (1999). Evolution of migration in a metapopulation. Bulletin of Mathematical Biology 61:531-550

Payne RJH \& Krakauer DC (1997). Sexual selection, space, and speciation. Evolution 51:1-9

Pimm ST (1979). Sympatric speciation: A simulation model. Biological Journal of the Linnean Society 11:131-139

Rand DA \& Wilson HB (1993). Evolutionary catastrophes, punctuated equilibria and gradualism in ecosystem evolution. Proceedings of the Royal Society of London B 253:137-141

Ridley M (1993). Evolution. Boston, MA, USA: Blackwell Scientific Publications

Rundle HD \& Schluter D (1998). Reinforcement of stickleback mate preferences: Sympatry breeds contempt. Evolution 52:200-208

Sætre GP, Moum T, Bures S, Kral M, Adamjan M \& Moreno J (1997). A sexually selected character displacement in flycatchers reinforces premating isolation. Nature 387:589-592

Schilthuizen M (2000). Ecotone: Speciation-prone. Trends in Ecology and Evolution 15:130-131

Schluter D (1996a). Ecological causes of adaptive radiation. The American Naturalist 148:S40S64

Schluter D \& Nagel LM (1995). Parallel speciation by natural selection. The American Naturalist 146:292-301

Schneider CJ, Smith TB, Larison B \& Moritz C (1999). A test of alternative models of diversification in tropical rainforests: Ecological gradients vs. rainforest refugia. Proceedings of the National Academy of Sciences of the USA 96:13869-13873

Seehausen O, van Alphen JJM \& Witte F (1997). Cichlid fish diversity threatened by eutrophication that curbs sexual selection. Science 277:1808-1811

Slatkin M (1980). Ecological character displacement. Ecology 61:163-177

Taper ML \& Case TJ (1985). Quantitative genetic models for the coevolution of character displacement. Ecology 66:355-371

Taper ML \& Case TJ (1992). Models of character displacement and the theoretical robustness of taxon cycles. Evolution 46:317-333

Taylor P (1989). Evolutionary stability in one-parameter models under weak selection. Theoretical Population Biology 36:125-143

Turner GF \& Burrows MT (1995). A model of sympatric speciation by sexual selection. Proceedings of the Royal Society of London B 260:287-292

Udovic D (1980). Frequency-dependent selection, disruptive selection, and the evolution of reproductive isolation. The American Naturalist 116:621-641

Van der Laan JD \& Hogeweg P (1995). Predator-prey coevolution: Interactions across different time scales. Proceedings of the Royal Society of London B 259:35-42 
Van Dooren TJM (1999). The evolutionary ecology of dominance-recessivity. Journal of Theoretical Biology 198:519-532

Van Doorn GS \& Weissing FJ (2001). Ecological versus sexual selection models of sympatric speciation. Selection 2:17-40

Van Tienderen PH \& de Jong G (1986). Sex ratio under the haystack model: Polymorphism may occur. Journal of Theoretical Biology 122:69-81

Vincent TL, Cohen Y \& Brown JS (1993). Evolution via strategy dynamics. Theoretical Population Biology 44:149-176

Wilson DS \& Turelli M (1986). Stable underdominance and the evolutionary invasion of empty niches. The American Naturalist 127:835-850

Wright S (1931). Evolution in Mendelian populations. Genetics 16:97-159

Wood CC \& Foote CJ (1996). Evidence for sympatric genetic divergence of anadromous and nonanadromous morphs of sockeye salmon (Oncorhynchus nerka). Evolution 50:12651279 